\title{
Convergence of 5G Technologies, Artificial Intelligence and Cybersecurity of Networked Societies for the Cities of Tomorrow
}

\author{
Trung Q. Duong ${ }^{1}$ • Van-Phuc Hoang ${ }^{2}$. Cong-Kha Pham ${ }^{3}$ \\ Published online: 11 May 2021 \\ (C) Springer Science+Business Media, LLC, part of Springer Nature 2021
}

As many as 100 billion devices will be connected to the Internet by 2025. It has been projected that the total volume of data traffic will nearly triple between 2016 and 2021, of which about $75 \%$ will originate from non-PC devices and about $42 \%$ of all connections will be for M2M communication between over 10 billion smart objects. It is anticipated that we will witness an up to 10,000 - fold growth in wireless data traffic by the year 2030. Predictions evidently indicates the skyrocketing demand on data traffic and applications for machine type communication such as self-driving vehicles, healthcare monitoring, smart cities and factories, and artificial intelligence- based personalized assistants along with traditional human-centric communications. Coexistence of human-centric and machine-type services as well as hybrids of these will make next generation wireless networks more diverse and complex.

Current wireless radio access techniques are not capable of delivering these new applications and may pose a much higher security risk than the $\mathrm{WiFi}$ and $4 \mathrm{G}$ networks did. Without novel approaches, mobile networks will grind to a halt unless more capacity is added to mobile networks. In order to better support the Internet-of-Things (IoT) applications, many technical challenges need to be overcome in $5 \mathrm{G}$ and beyond including network architectures, network resource allocation schemes, and advanced signal processing techniques, etc. On the other hand, in IoT systems, the hardware security assurance is a critical and emerging issue. It is reported that about $70 \%$ of IoT devices are vulnerable to cyber-attacks. In

Trung Q. Duong

trung.q.duong@gmail.com

1 Queen's University Belfast, University Rd, Belfast BT7 1NN, UK

2 The Department of Microelectronics, Le Quy Don Technical University, Hanoi, Vietnam

3 Department of Computer and Network Engineering, The University of Electro-Communications, Tokyo, Japan addition, deep learning and AI techniques have been considered as promising approaches to unleash the full potential of $5 \mathrm{G}$ networks, IoT and cyber-physical systems.

To overcome the aforementioned challenges of emerging issues for networks of future, this special issue focuses on (but are not restricted to) the following topics: Advanced network architecture design for IoT towards 5G; IoT applications to disaster management, smart cities, smart environment, and smart agriculture; Energy-efficiency in 5G for IoT applications; 5G wireless heterogeneous networks: design and optimization; Mobility management of $5 \mathrm{G}$ networks for IoT applications; $5 \mathrm{G}$ wireless communications and networks for surveillance and management; 5G technologies: NOMA, fullduplex, massive MIMO, network planning, mmWave, URLLC; Big data and IoT data analytics; Security and privacy concerns in 5G wireless communications; Hardware forensics; Deep learning for hardware oriented cybersecurity; Machine learning for resource allocation in wireless networks; Emerging memory and computing technologies for future networks; and Advanced signal processing for future networks.

The special issue includes five high-quality papers. In the first paper entitled "Energy Efficiency of Full-Duplex Cognitive Radio in Low-Power Regimes under Imperfect Spectrum Sensing", the authors investigate the energy efficiency (EE) of a full duplex (FD) cognitive radio (CR) system, in which the low-power regime is considered under a practical self-interference cancellation performance and imperfect spectrum sensing. In the system, the secondary user (SU) is equipped with the capacity of self-interference suppression (SIS), i.e., in an opportunistic spectrum access network. In other words, the SU can work in simultaneous transmit and sense (TS) mode to increase the channel sensing quality. By using TS mode, the sensing performance of the FD CR networks evaluated by false-alarm and miss-detection probabilities is studied and compared to the conventional half duplex (HD) CR networks using transmit only mode (TO). The results show that the Gaussian-mixture channel, which is widely used to capture the asynchronism in heterogeneous cellular 
networks, can be applied to the channel of the secondary network well in imperfect spectrum sensing scenario. Furthermore, the low-signal-to-noise-ratio (low-SNR) metrics of the minimum energy per bit and the wideband slope of the spectral efficiency curve are analytically characterized to derive the closed-form fundamental limits. This way enables us to identify the practical signaling strategies for optimal efficiency of the low-SNR regime in FD CR system. Finally, the benefit of the FD CR system over the HD CR one is demonstrated in terms of EE.

The second paper is about "Evaluating the Performance of Full-Duplex Energy Harvesting Vehicle-to-Vehicle Communication System over Double Rayleigh Fading Channels". In this paper, the authors consider the vehicle-tovehicle $(\mathrm{V} 2 \mathrm{~V})$ communication system. The system is employed by full-duplex (FD) and energy harvesting (EH) techniques. All the source, relay and destination nodes are moving in the context of $\mathrm{V} 2 \mathrm{~V}$ communications. The source and the relay nodes can harvest the radio energy from a power beacon (PB) for data transmission. The exact expressions of the outage probability (OP) and symbol error probability (SEP) are derived to investigate the impacts of various parameters on the system performance. Numerical results show that the system performance is strongly impacted by the number of transmission antennas of the $\mathrm{PB}$, the EH duration, the residual self-interference, and the transmission distances. In addition, there also exists an optimal EH duration and optimal distance between the source and relay nodes that can provide the best system performance. Monte-Carlo simulations are done to validate all theory analysis.

In the third paper, the authors study "A Reliable Linkadaptive Position-based Routing Protocol (RLPR) for Flying Ad hoc Network (FANET)". To develop the conventional routing protocols of FANET in a simple network environment, i.e., using a minimum hop count criterion to find the best route between a source and a destination, the authors utilize relative speed, signal strength, and energy of the nodes along with the geographic distance towards the destination using a forwarding angle. This angle is to determine the forwarding zone that decreases the undesirable control messages in the network for route discovery. The RLPR can provide a better network performance by selecting not only the relay nodes that are in the forwarding zone towards the destination, but also the next hop with proper energy level, signal strength, and relative speed of the nodes for a higher connectivity level. The simulation results performed by network simulator (NS-2.35) are shown to investigate the benefits of the proposed RLPR over the ad hoc on-demand distance vector and the robust and reliable predictive based routing protocols in terms of control overhead message, lifetime, and search success rate.

A clustering method for table structure recognition in scanned images (ClusTi) is proposed in the fourth paper. It is challenging in optical character recognition (OCR) for scanned paper invoices due to the variability of 19 invoice layouts, different information fields, large data tables, and low scanning quality. The table structure recognition plays an important role in accurately positioning and extracting the rows, columns, and cells. In this paper, the authors propose the ClusTi method for the recognition of the structure of tables in invoice and scanned images that can overcomes the problems of existing methods such as DeepDeSRT, i.e., only deal with high-quality born-digital images (e.g., PDF) with low noise and apparent table structure. To do so, the ClusTi first removes the heavy noises from the table images using a clustering algorithm. It then extracts all the text boxes by using state-of-the-art text recognition. Finally, based on the horizontal and vertical clustering algorithm with optimized parameters, the proposed method groups the text boxes into their correct rows and columns, respectively. The results show that the CluSTi achieves an F1-score of 87.5\%, 98.5\%, and 94.5\%, respectively. It also outperforms the DeepDeSRT with an F1score of $91.44 \%$ on only 34 images from the ICDAR 2013 competition dataset.

Finally, the last paper is about "Meteorological and Hydrological Drought Assessment for Dong Nai River Basin, Vietnam under Climate Change". In this paper, the authors examine future changes in meteorological, hydrological drought under the impact of climate change in Dong Nai River Basin, using Standardized Precipitation Index (SPI) and Stream flow Drought Index (SDI). The Soil and Water Assessment Tool (SWAT) is used estimate the stream flow in baseline (1980-2005) and the climate change (RCP 4.5, 2016-2035) scenarios for meteorological and hydrological calculation. The results show that 1) both types of drought tend to occur in the dry season, 2) the area is affected by meteorological and hydrological drought expanding in both baseline and RCP 4.5 scenarios, and 3) meteorological drought duration is also significantly increased, especially severely drought months. Although it slightly decreases in the hydrological drought duration, the number of months in moderately drought in the sub-basins still goes up in the climate change scenario. The findings could be useful for water shortage assessment and allocation planning in the context of climate change in the Dong Nai River Basin.

Publisher's note Springer Nature remains neutral with regard to jurisdictional claims in published maps and institutional affiliations.

Trung Q. Duong is a Professor and Chair of Telecommunications at Queen's University Belfast, UK. He is also holding a prestigious Research Chair of Royal Academy of Engineering. His current research interests include wireless communications, signal processing, machine learning and data analytics. He is an author/co-author of more than 300 technical papers (including 175 ISI journals and 128 conference papers). 
Dr. Duong is currently serving as an Editor for IEEE Trans on Wireless Communications. He was an Editor of IEEE Trans on Communications, IEEE Communications Letters, IET Communications. He has also served as the Guest Editor of the special issue (SI) for the following journals: Lead Guest Editor of the IEEE Journal in Selected Areas on Communications (JSAC) for the SI on "Location Awareness for Radios and Network" in 2015; Lead Guest Editor of the IET Communications for the SI on "Secure Physical Layer Communications" in 2013 and on "Green Telecommunications Systems and Computing" in 2016; Guest Editor of the IEEE Wireless Communications Magazine for the SI on "Green Media: The Future of Wireless Multimedia Networks" in 2014; Guest Editor of the IEEE Communications Magazine for the SI on "Millimeter Wave Communications for 5G" in 2014 and "Energy Harvesting Communications" in 2015; Guest Editor of the IEEE Access for the SI on "Security for Wireless Communications and Networking" in 2016 and on "Exploiting the Benefits of Interference in Wireless Networks: Energy Harvesting and Security" in 2017; Guest Editor of the EURASIP Journal on Wireless Communications and Networking for the SI on "Cooperative Cognitive Networks" in 2013; Guest Editor of the EURASIP Journal on Advances Signal Processing for the SI on "Security Challenges and Issues in Cognitive Radio Networks" in 2014; Editor for Wiley Transactions on Emerging Telecommunications Technologies (2013-2016) and Electronics Letters (2015-2016). He was awarded the Best Paper Award at the IEEE Vehicular Technology Conference (VTC-Spring) in 2013, IEEE International Conference on Communications (ICC) 2014, and IEEE Global Communications Conference (GLOBECOM) 2016.

Van-Phuc Hoang received $\mathrm{PhD}$ degree in Electronic Engineering from The University of Electro-Communications, Tokyo, Japan in 2012. He is currently an Associate Professor with the Faculty of Radio-Electronic Engineering and also as Deputy Head of The Department of
Microelectronics, Le Quy Don Technical University, Hanoi, Vietnam. He was a postdoc researcher, then an invited scholar with The University of Electro-Communications, Tokyo, Japan in 2013, 2015 and 2017. He was also an invited scholar with University of Strathclyde, UK in 2014 and with Telecom ParisTech, France in 2018. His research interests include digital circuits and systems, low power IC design, hardware security, embedded systems and VLSI architecture for digital signal processing. He has authored or co-authored in more than 50 papers published in international journals and conference proceedings. He has served as Technical Programme Chair for IEEE international conferences such as IEEE 12th International Symposium on Embedded Multicore/Many-core Systems-on-Chip (MCSoC2018) and The 3rd International Conference on Recent Advances in Signal Processing, Telecommunications \& Computing (SigTelCom2019). He has also served as a reviewer for many top-tier international journals microelectronics, hardware security and embedded systems, and has been a Technical Programme Committee Member for several flag-ship international conferences in the related fields.

Cong-Kha Pham received the B.S., M.S., and Ph.D. degrees in electronics engineering from Sophia University, Tokyo, Japan in 1989, 1990 and 1992, respectively. He is currently a Professor with the Department of Computer and Network Engineering, The University of ElectroCommunications, Tokyo, Japan. His research interests include hardware security, open source processors and the design of analog/digital systems using integrated circuits. He has authored or co-authored in over 180 papers published in international journals and conference proceedings. Prof. Pham received several best conference paper awards. He has served as a reviewer for many top-tier international journals on VLSI systems and integrated circuits, and has also been a Technical Programme Committee Member for several flag-ship international conferences in the related fields. 